\title{
NEUROCIÊNCIA COGNITIVA COMO BASE PARA ANÁLISE DO PROCESSO DO PENSAMENTO COMPUTACIONAL, ATRAVÉS DA PROGRAMAÇÃO
}

\author{
Lucas Tadeu Hinterholz ${ }^{1}$ \\ Cristiane Goulart Pinto ${ }^{2}$ \\ Guilherme Machado Düren ${ }^{3}$ \\ Thiago Rebelatto Oliveira 4 \\ Samanta Ghisleni Marques ${ }^{5}$ \\ Werner Haetinger ${ }^{6}$ \\ Marcia E. J. Kniphoff da Cruz ${ }^{7}$
}

\section{RESUMO}

Os estudos no campo da Neurociência Cognitiva estão evoluindo e apontando-a como essencial para o entendimento da construção do conhecimento. Esta pesquisa faz parte da proposta do projeto Contribuição da Tecnologia Computacional para a Assistência Social e apoio do projeto de extensão Unisc - Inclusão Digital, desenvolvidos na Universidade de Santa Cruz do Sul e executados por docentes e estudantes do curso de Licenciatura em Computação. Como base teórica foram estudados conceitos da Neurociência Cognitiva e Abstração Reflexionante para a compreensão dos processos mentais e acompanhamento de resultados de aprendizagem do estudante, enquanto utiliza computador para programar em uma linguagem específica. A Metodologia contou com um Estudo de Caso, desenvolvido através de oficina de ensino de programação para adolescentes entre 14 e 17 anos, os quais não possuíam qualquer conhecimento prévio. Estes responderam a um teste do Método Clínico Piagetiano - MCP relacionado à memória implícita e explícita, adaptado para aplicação durante a utilização de linguagem de programação. Como resultado, observou-se que 83,34\% dos participantes correspondeu à expectativa e apresentou características de abstração e consciência compatíveis com o nível cognitivo reflexivo. Neste sentido, afirma-se que a programação computacional é incentivo à cognição humana, contribui para o desenvolvimento do Pensamento Computacional, devendo ser inserida no ensino formal junto aos currículos escolares.

Palavras-chave: Programação. Pensamento Computacional. Neurociência. Abstração.

\section{ABSTRACT}

Studies in the field of Cognitive Neuroscience are evolving and indicating it to be essential for an understanding of knowledge building. This research is part of the proposal of the project "Contribution of Computational Technology for Social Assistance" and as support the

${ }^{1}$ Acadêmico do Curso de Licenciatura em Computação da Universidade de Santa Cruz do Sul - UNISC. <lhinterholz@gmail.com>

2 Acadêmica do Curso de Licenciatura em Computação da Universidade de Santa Cruz do Sul - UNISC. <crica.goulart@gmail.com>

${ }^{3}$ Acadêmico do Curso de Licenciatura em Computação da Universidade de Santa Cruz do Sul - UNISC. <guilhermeduren@yahoo.com.br>

${ }^{4}$ Mestrando do Programa de Pós-Graduação de Sistemas e Processos Industriais - Universidade de Santa Cruz do Sul - UNISC. <rebelattonext@gmail.com>

${ }^{5}$ Aluna egressa do Curso de Licenciatura em Computação da Universidade de Santa Cruz do Sul - UNISC <samantaghisleni@gmail.com>

${ }^{6}$ Departamento de Informática da Universidade de Santa Cruz do Sul - UNISC. <werner@ unisc.br〉

${ }^{7}$ Departamento de Informática da Universidade de Santa Cruz do Sul - UNISC. <俩cruz@unisc.br> 
extension project "Unisc - Digital Inclusion", developed at the University of Santa Cruz do Sul and performed by teachers and undergraduate students of Computer Science Degree. For theoretical basis were studied concepts of Cognitive Neuroscience and Reflexive Abstraction in order to understand the mental processes and to follow the results of the student learning while using computer to program in a specific language. The methodology included a Case Study, developed through the programming teaching workshop for teenagers aged 14 to 17 years old, who did not have any prior knowledge. They answered a Piagetian Clinic Method test related to implicit and explicit memory and adapted for when using a programming language. As a result, it was observed that $83,34 \%$ of the participants corresponded to the expectation and showed characteristics of abstraction and awareness compatible with reflective cognitive level. In this sense, it is said that programming in computational media is encouraging to human cognition contributes to the development of computational thinking and should be included in formal education, along with the high school curriculums.

Keywords: Programming. Computational Thinking. Neuroscience. Abstraction.

\section{INTRODUÇÃO}

A aprendizagem continua sendo o objetivo principal das escolas de todos os níveis de ensino e hoje elementos tecnológicos, mais especificamente objetos computacionais $^{8}$ são incorporados às rotinas escolares e acadêmicas para o sucesso em todas as áreas do conhecimento. Em geral, a programação de computadores é considerada de difícil aprendizado, contudo, pode vir a ser introduzida já no Ensino Fundamental se encaminhada com uma consistente base teórica e metodologia apropriada (CRUZ, 2007; CRUZ, 2008; CRUZ, 2009a; CRUZ, 2009b). Neste sentido, o projeto de pesquisa intitulado Contribuição da Tecnologia Computacional para a Assistência Social teve como objetivo principal atender a Tipificação Nacional de Serviços Socioassistenciais, relativa à Resolução No. 109, de 11 de Novembro de 2009 em parceria com o projeto de extensão UNISC - Inclusão Digital. Foram oferecidas oficinas de informática à população com alto índice de vulnerabilidade social e ao público em geral. Elencou-se em um de seus eixos o estudo da Neurociência Cognitiva e da Abstração Reflexionante como bases teóricas para as práticas metodológicas no ensino da programação. Dentre várias oficinas oferecidas, uma em especial foi utilizada na coleta de dados e desenvolvida com adolescentes que responderam ao teste Piagetiano (PIAGET, 1995).

Segundo Damásio (1996), a razão humana não está relacionada a um único centro

\footnotetext{
${ }^{8}$ Objetos computacionais compreendem software e hardware ou programas de computador e equipamentos que possam auxiliar na aprendizagem de determinados conteúdos ou habilidades.
} 
cerebral, mas sim a vários sistemas cerebrais dentro de muitos níveis de organizações neurais. As emoções, sentimentos e regulação biológica têm papel fundamental na razão humana. $\mathrm{O}$ organismo humano é a referência de base para as interpretações que as pessoas fazem do mundo e a construção do sentido de objetividade é parte essencial das experiências vividas. Com este propósito, foi utilizado o Método Clínico Piagetiano - MCP como metodologia da pesquisa, o que contribui para que os pesquisadores acompanhassem o processo cognitivo ou mental com o qual os sujeitos interpretam o mundo e constroem seu sentido de objetividade. Buscando atender esta finalidade, a pesquisa incentivou a aproximação de bases teóricas da Neurociência Cognitiva como aporte ao aprendizado de programação e considerou que a programação computacional é incentivo à cognição humana.

\section{NEUROCIÊNCIA COGNITIVA}

A Neurociência Cognitiva, segundo Tabacow (2007), é um campo da ciência que estuda a aquisição de conhecimento e o comportamento em sua origem. É considerada uma linha de estudo relativamente nova. Especificamente, segundo Kandel (neurobiólogo, psiquiatra) e colaboradores, citado por Tabacow (2007, p. 14): "um misto de neurofisiologia, anatomia, biologia desenvolvimentista, biologia celular e molecular, psicologia cognitiva e ciência computacional". Segundo Kandel et al. (1997) pesquisas experimentais tentam relacionar mecanismos moleculares à cognição, sendo que "cognição" pode ser definida como "Função da inteligência ao adquirir um conhecimento." (PRIBERAM, s/p) ${ }^{9}$ Para Kandel et al., "[...] a fusão da Biologia com a Psicologia Cognitiva é mais do que uso comum de métodos e de conceitos" (1997, p. 552).

Amplia-se esta afirmação quando é possível contar com a Epistemologia Genética de Jean Piaget para encaminhar novos estudos e mais especificamente a Abstração Reflexionante. Por sua vez, a Psicologia Cognitiva atua no estudo e compreensão da memória, linguagem e percepção. Este braço da Ciência Cognitiva apresenta, como característica, o estudo comportamental do indivíduo, sendo este o principal aspecto que a diferencia da Neurociência Cognitiva. Esta, por sua vez, faz uma abordagem, mais biológica e neural da cognição e dos processos mentais.

\footnotetext{
${ }^{9}$ Definição “cognição” - Dicionário Priberam. <http://www.priberam.pt/dlpo/default.aspx?pal=cognicao>.
} 
Um dos aspectos principais da Psicologia Cognitiva é a abordagem do processamento da informação, que prevê a comparação dos processos mentais com os processos computacionais. Sendo assim, segundo Matlin (2004, p. 9), pode-se representar um determinado processo cognitivo por informações que passam por etapas, semelhante ao processamento de um programa de computador, por exemplo. Sob esta sugestão, o cérebro, para chegar a determinada conclusão ou resposta, passaria por vários subprocessos de maneira lógica (se x, então y; enquanto x, faça y), tal como acontece nos algoritmos em Computação. Outros alvos de investigação da Psicologia Cognitiva envolvem a atenção, o reconhecimento, a percepção e a tomada de decisão.

A partir de 1960 que a Neurociência Cognitiva ampliou sua abordagem integrando cinco áreas: a eletro-fisiologia (estuda o comportamento do neurônio face a um estímulo), reconhecimento de grupos celulares (neurônios, relacionados especificamente aos processos cognitivos superiores como a atenção e a tomada de decisão), neurociência e sistemas de psicologia cognitiva (a cognição é constituída por vários sistemas cognitivos); técnicas de imagem radiológica (neuroimagem: mostram a relação de neurônios com atos mentais específicos), ciência computacional e psicofísica (relação entre atributos físicos de um estímulo e a percepção) (TABACOW, 2007). Os estudos começaram a florescer na década de 1980 quando neurocientistas e psicólogos cognitivistas passaram a empregar técnicas de imagem cerebral para registrar a atividade do cérebro (MATLIN, 2004). Este campo de estudo parte do princípio de que, para compreender como as pessoas se comportam, pensam, sentem e agem, é necessário conhecer de que forma as ações integradoras do encéfalo, como a atividade simultânea de um conjunto de neurônios, produz a cognição. Os estímulos desencadeiam atividade neuronal e o que acontece após é foco do estudo, avaliando também qual será a resposta do indivíduo ao processo. Estes conhecimentos fornecem base para quem procura ocupar as informações científicas para a evolução cognitiva (TABACOW, 2007).

As funções cognitivas altamente complexas estão localizadas em regiões específicas do cérebro, mas não significa que apenas uma região seja responsável, exclusivamente, por determinada função. Significa que certas regiões cerebrais estão mais envolvidas com um tipo de função que outras. Os três principais córtices de associação são o pré-frontal, parietaltemporal-occipital e áreas de associação límbica (KANDEL et al., 1997).

A Neurociência Cognitiva ou Neurocognição prioriza estudos sobre pacientes com lesões cerebrais e com imagens que representam a atividade cerebral, indicando regiões com maior fluxo sanguíneo enquanto pessoas leem, falam ou praticam outras atividades. Alguns fatores são muito evidenciados como as percepções e a memória, e afirmam a distinção entre 
"mente e cérebro". Considera o avanço da Computação um marco para os estudos mais recentes e com melhores resultados, enquanto a área da Inteligência Artificial (subárea da Computação) busca assemelhar o funcionamento dos computadores aos dos seres humanos, valendo-se dos estudos da Neurociência e Psicologia Cognitiva. Assim, importa saber: "o aprendizado pode causar alterações estruturais no cérebro." Experimentos com animais têm confirmado esta proposição, ou seja, o aprendizado produz alterações funcionais e estruturais em células neurais específicas. Nos humanos cada componente funcional é representado por centenas de milhares de células nervosas. O aprendizado, provavelmente, causa alteração de muitas células nervosas e possivelmente no padrão de interconexão dos diversos sistemas sensoriais e motores participantes de determinada tarefa aprendida (KANDEL et al., 1997).

As funções da memória são executadas em regiões específicas do cérebro, como nos lobos temporais. A memória é dividida em implícita ou explícita. A explícita depende do lobo temporal medial, codifica a informação sobre eventos autobiográficos e conhecimento de fatos e envolve processos cognitivos como avaliação, comparação e inferência. A implícita não exige recordação deliberada e depende de diversas vias perceptivas e reflexas não podendo, em condições usuais, ser expressa por palavras.

A mente não é o cérebro para Pinker (1998), mas o que o cérebro faz e, enquanto confirma a importância da pesquisa de Darwing, afirma:

\begin{abstract}
A teoria computacional da mente [...]. Ela afirma que crenças e desejos são informações, encarnadas como configurações de símbolos. Os símbolos são os estados físicos de bits de matéria, como os chips de computador ou neurônios do cérebro. Eles simbolizam coisas do mundo porque são desencadeados via órgãos dos sentidos e devido ao que fazem depois de ser desencadeados. Se os bits de matéria são ajustados para topar com outros bits de matéria que constituem outros símbolos exatamente do jeito certo, os símbolos correspondentes a uma crença podem originar outros símbolos correspondentes a outra crença e assim por diante (p. 35-36).
\end{abstract}

Sendo imprescindível analisar em quais aspectos convergem a Neurocognição e a Abstração Reflexionante, considerando seu impacto para o desenvolvimento do Pensamento Computacional, as atividades desenvolvidas nesta pesquisa buscaram entender como a Neurociência Cognitiva pode auxiliar na compreensão do processamento cerebral e na coordenação das ações mentais, enquanto os participantes de oficina programam a Plataforma Arduino ${ }^{10}$ e empregam Abstração Reflexionante.

\footnotetext{
${ }^{10}$ Maiores informações sobre a placa Arduíno podem ser encontradas em sua homepage. Disponível em: http://www.arduino.cc/
} 


\section{ABSTRAÇÃO REFLEXIONANTE}

A abstração é fundamental ao processo de cognição humana e conta, inicialmente, com a abstração empírica, que se apoia no uso de objetos físicos para chegar à abstração reflexionante. Para distinguir a abstração reflexionante da abstração empírica, Piaget utilizouse de várias técnicas e procedimentos, valendo-se de materiais concretos como blocos até cálculos lógico-aritméticos, todos necessários para aplicação do $\mathrm{MCP}$, que prevê muita fidedignidade por parte do pesquisador. O MCP consiste em conversar com crianças ou adolescentes, a partir de questões semiestruturadas, observando suas reações e comportamento ao responder. Durante a execução das perguntas o examinador pode analisar as respostas dadas pelo sujeito e, dependendo do que foi dito, reelaborar questões e as empregar novamente, tendo o cuidado de não direcionar o pensamento ou raciocínio do entrevistado. Pode, ainda, formular novas questões fazendo com que reflita sobre o que respondeu. A análise final das respostas deve ser realizada após encerrar a aplicação do método (PIAGET, 2005).

Os estudos de Piaget, que resultaram na obra “Abstração Reflexionante”, apresentam consistente explanação teórica e relato minucioso de 18 técnicas ou procedimentos, detalhando a ação de crianças e adolescentes sobre os objetos em situações de experimento. Nestes experimentos o pesquisador apresenta, em etapas, duas ou mais técnicas (atividades com objetos) e ao final de cada etapa solicita que as crianças e adolescentes relatem o que realizaram. Para a conclusão são questionadas sobre o que há de semelhante entre as etapas das técnicas (salvo no experimento realizado com bebês), conforme o exemplo da inversão das operações aritméticas (PIAGET, 1995).

A técnica objetiva analisar a reversibilidade das operações aritméticas a partir do ponto de vista da abstração, numa situação que pareça um jogo. É desenvolvida em três etapas: primeira, construção e demolição de um "cogumelo" de sete peças; segunda, construção e demolição de um grande cubo, utilizando oito pequenos cubos; terceira, consiste em um problema de cálculo, em que pede-se ao sujeito que escreva um número qualquer em uma folha e, em seguida, acrescente o valor 3 a este número, multiplique por dois e, por fim, acrescente o valor 5. O pesquisador, então, pergunta qual foi o resultado e, através do cálculo inverso, fala qual foi o número que o sujeito escreveu no início do procedimento. Ao final de cada técnica, deve-se perguntar sobre a ordem utilizada para montar e desmontar o "cogumelo" e o cubo e solicitar uma comparação. Sobre o cálculo, é questionado se o conhecimento do resultado é necessário para reencontrar o número original e o porquê e sobre a necessidade de 
ordem na realização das operações ou não. Ao final, é solicitado ao sujeito que compare as três técnicas, dizendo se há ou não algo de semelhante entre elas.

A fala ou relato das crianças e adolescentes oportuniza ao pesquisador identificar o Estádio ou Nível em que cada um se encontra, sendo eles: Estádio I - correspondências globais, abstrações empíricas (ou pseudo-empíricas), Nível A1 - aparecem abstrações reflexionantes ativas, mas controladas por abstrações pseudo-empíricas; Nível A2 - permite comparação correta, mas sem "reflexão" nova sobre este "refletido". Estádio II, Nível B - a abstração reflexionante impõe-se a tal ponto que o sujeito deforma a metade dos fatos, reprimindo os controles da abstração pseudo-empírica e refletida; Estádio III - a abstração refletida se desdobra em uma reflexão sobre esta reflexão, permitindo ao sujeito livrar-se da incompatibilidade das conservações anteriores (PIAGET, 1995).

O processo de abstração reflexionante comporta dois aspectos inseparáveis: o reflexionamento, "projeção sobre um patamar superior daquilo que foi retirado do patamar inferior"; e a reflexão, "ato mental de reconstrução e reorganização sobre o patamar superior daquilo que foi assim transferido do inferior" (PIAGET, 1995, p. 274-275). A união da reflexão e do reflexionamento é formadora dos patamares sucessivos e não somente fonte de passagens de um para outro. Dessa forma, a reflexão reorganiza as informações geradas a partir do reflexionamento, produzindo uma nova construção do conhecimento. Este processo não possui início ou fim, forma um ciclo que só é possível através da coordenação de ações cerebral humana. Para Piaget (1995):

[...] todo reflexionamento de conteúdos (observáveis) supõe a intervenção de uma forma (reflexão), e os conteúdos assim transferidos exigem a construção de novas formas devido à reflexão. Há, assim, pois, uma alternância ininterrupta de reflexionamentos $\rightarrow$ reflexões $\rightarrow$ reflexionamento; e (ou) de conteúdos $\rightarrow$ formas $\rightarrow$ conteúdos reelaborados $\rightarrow$ novas formas, etc., de domínio cada vez mais amplo, sem fim e, sobretudo, sem começo absoluto (p. 276).

Os patamares do reflexionamento então, de modo geral, iniciam no nível sensóriomotor, sendo o primeiro a ação que conduz a uma representação. O segundo patamar é o da reconstituição da sequência das ações, reunindo as representações em um todo coordenado. $\mathrm{O}$ terceiro patamar é o da comparação, em que a ação total é comparada a outras análogas ou diferentes. Estas comparações destacam estruturas comuns e não comuns, gerando o quarto patamar, caracterizado pelas reflexões sobre as reflexões precedentes, chegando a vários graus de "meta-reflexão" ou pensamento reflexivo, que permite ao sujeito encontrar as razões das conexões que, até o momento, eram simplesmente constatadas (PIAGET, 1995).

Dessa forma, para Piaget (1995, p. 205), “a abstração reflexionante é a fonte contínua 
de novidades, porque atinge novas 'reflexões' sobre cada um dos planos sucessivos do 'reflexionamento' e estes se engendram sem que sua sequência seja jamais acabada'. Considera-se que as novas reflexões e reflexionamentos desencadeiam as habilidades para o Pensamento Computacional.

\section{PENSAMENTO COMPUTACIONAL}

Pensamento computacional é uma habilidade fundamental para todos, não apenas para cientistas da Computação, e envolve a solução de problemas projetando sistemas conforme a compreensão humana, inspirando-se em conceitos fundamentais da Ciência da Computação. Pensamento Computacional inclui uma variedade de ferramentas mentais que refletem a amplitude do campo da Ciência da Computação. Ao resolver um problema particular pode-se perguntar: qual a maneira mais fácil de resolução? Qual é a melhor maneira de resolução? A informática repousa sobre sólidas bases teóricas para responder a tais perguntas.

Conforme Ribeiro (2012), o Pensamento Computacional oportuniza pensar e agir no sentido de como resolver problemas e quais equipamentos (computadores) corretos utilizar para esta resolução, desenvolvendo algoritmos que podem ser expressos em diferentes níveis de abstração. Para que este pensamento seja introduzido na Educação Básica é imprescindível que, já nos anos iniciais do Ensino Fundamental, sejam utilizadas linguagens de programação que fomentam o Pensamento Computacional, embora não sejam responsáveis pelo seu desenvolvimento. Nunes (2014) exemplifica esta questão com a seguinte comparação: para aprender a Língua Portuguesa não é necessário aprender a utilizar o Word ou outro editor de textos e para aprender Matemática não é necessário aprender a utilizar o Excel ou outra planilha eletrônica. Todo ser humano possui pensamento lógico, porém em diferentes níveis que podem ser aprimorados, auxiliando os processos de tomada de decisão. O pensamento lógico leva a um processo de resolução de problemas baseado em etapas com resultados que podem servir para a decisão/resolução de etapas subsequentes.

Segundo Nunes (2014), a função do computador é a de computar termos, não importando seu tipo: números, textos, imagens, sons etc. A computação de termos, como dados de entrada, fornece termos, como dados de saída e afirma, portanto: 
computação. Conclui-se que todos os alunos das universidades deveriam aprender uma introdução à Ciência da Computação, que os capacitasse a identificar os problemas da área que poderiam ser solucionados por métodos desenvolvidos pela computação, bem como a descrever as soluções, usando uma linguagem científica apropriada e simples. Noções como as de Algoritmos, Complexidade Computacional, Organização de Computadores, Linguagens de Programação, Redes de Computadores, Banco de Dados, Sistemas Operacionais são fundamentais para que os futuros sociólogos, economistas, músicos possam interagir com profissionais de computação, desenvolvendo um pensamento interdisciplinar (3) além de, como cidadãos, adquirir conhecimentos sobre este importante e novo ramo da ciência (s. p.).

Neste sentido, entende-se que os adolescentes podem e devem iniciar a experiência com o desenvolvimento de Algoritmos, utilizando linguagem de programação acessível, através da qual se possa observar resultados físicos como o acionamento de pequenas luzes denominadas Light Emitting Diode - LEDs e motores através da Plataforma Arduino e que é possível analisar o processo cognitivo que cada estudante percorre para alcançar a reflexão e a consciência.

\section{METODOLOGIA}

A pesquisa contou com metodologia Qualitativa para aferir resultados de aprendizagem com estratégias de Estudo de Caso envolvendo aplicação de métodos com base na Neurociência Cognitiva para acompanhamento do processo cognitivo de desenvolvimento do Pensamento Computacional. Caracterizou-se como um estudo transversal, sendo que, contou com análises teóricas, levantamento de casos correlatos e a proposta de métodos, estratégias ou técnicas para acompanhamento do processo cognitivo da formação do Pensamento Computacional. A metodologia também foi observacional durante entrevista e aplicação do MCP e de intervenção no momento em que foram oferecidas oportunidades nos Laboratórios de Informática da UNISC e acesso à Linguagem "C", considerada uma das mais populares linguagens de programação, e à Plataforma Arduino. Foi utilizado o Método Clínico Piagetiano - entrevistas com os participantes, envolvendo coleta e análise de dados, com os quais se acompanhou o pensamento, sem interferir nas respostas, elaborando sempre novas perguntas a partir das respostas e avaliando a qualidade e abrangência destas.

A pesquisa assumiu caráter aplicado, tendo como meta, em concordância a Cervo (2007), contribuir para fins práticos, buscando soluções para problemas concretos e transformando em ação concreta os resultados do trabalho. Foi estabelecido um plano de análise qualitativo quando relacionado aos ganhos de aprendizagem pelos beneficiados, no 
emprego do método especificado.

O público alvo envolveu beneficiários do Abrigo Municipal de Santa Cruz do Sul e do projeto de extensão UNISC - Inclusão Digital e especificamente na oficina desenvolvida, foi oportunizada a programação da Plataforma Arduino, através da qual os estudantes desenvolveram junto ao computador listas de instruções para acionamento de motores e LEDs conectados à Plataforma a partir da linguagem de programação "C". Da oficina participaram 12 adolescentes entre 14 e 17 anos que não possuíam qualquer conhecimento prévio sobre programação. No primeiro encontro foi aplicado teste escrito para verificação da atenção e da lógica de pensamento de cada participante. Durante o desenvolvimento da oficina, através de entrevistas individuais, os participantes responderam ao teste Piagetiano - MCP (PIAGET, 1995). Durante a aplicação do MCP foram realizadas anotações sobre a evocação da memória implícita e explicita, conforme descrito na seção quatro (4). De acordo com Piaget (1971), o conhecimento resulta de interações que são produzidas como resultante da ação do sujeito sobre o objeto. Da mesma forma, na perspectiva de Damásio (2000), o conhecimento se dá através da consciência que consiste em construir um conhecimento sobre dois fatos: um organismo está empenhado em relacionar-se com algum objeto, e o objeto nessa relação causa uma mudança no organismo.

\section{RESULTADOS}

O teste solicitou a ordenação de passos de várias tarefas indicando que 83,34\% dos participantes possuía lógica operatória de pensamento e patamar muito bom de abstração reflexionante. Contudo, 16,66\% dos integrantes apresentou resultados de aprendizagem condizentes apenas com o Nível IIA, no qual aparecem abstrações reflexionantes ativas, mas controladas por abstrações pseudo-empíricas, ou seja, necessita de exemplos práticos e manipuláveis para dar encaminhamento à abstração.

Ainda, conforme Piaget (2005) os entrevistados apresentam contextos mentais que podem ser de não-importismo, fabulação ou brincadeira, crença sugerida, crença desencadeada e crença espontânea. Estes cinco contextos do MCP comparados à teoria de evocação da memória explícita, foram relacionados da seguinte forma: (a) não-importismo e fabulação ou brincadeira: não evoca a memória explícita; (b) crença sugerida: evoca parcialmente a memória explícita; (c) crença desencadeada e crença espontânea: evoca com agilidade a memória explícita. 
Como resultado observou-se que $83,34 \%$ dos participantes correspondeu à expectativa e apresentou plena coordenação de ações - pensamento lógico/abstração, sendo que a porcentagem de sucesso foi ampliada em $6 \%$ do início para o final da oficina. Os resultados de aprendizagem enfatizaram emprego adequado da memória explícita e características próprias do Estádio III, no qual a abstração refletida se desdobra em uma reflexão sobre esta reflexão, permitindo ao sujeito, neste Estudo de Caso, dissipar as dúvidas e desenvolver o Algoritmo na linguagem "C" com fluência e agilidade mental.

\section{DISCUSSÕES E CONCLUSÃO}

A programação na Linguagem "C" permitiu a visualização de resultados físicos que geraram um retorno imediato pelo acionamento de motores e LEDs junto à Plataforma Arduino e consequentemente, um ambiente ideal para as análises propostas nesta pesquisa. Foi possível aferir que o aprendizado sobre o desenvolvimento de Algoritmos auxilia a aprendizagem e incentiva o Pensamento Computacional, ou seja o pensamento reflexivo. Os beneficiários tiveram a oportunidade de experimentar, discutir e agir sobre a programação e sobre a Plataforma Arduino. Complementando, observou-se um efetivo envolvimento dos estudantes com a linguagem de programação através da definição de comandos para programação do kit. Considera-se, portanto, a programação um instrumento de apoio para a coordenação de ações e para a abstração cognitiva necessária aos aspectos relacionados à programação computacional. $\mathrm{O}$ envolvimento do grupo foi satisfatório e a maioria dos adolescentes demonstrou facilidade ao programar, evocando a memória explícita com agilidade.

Como continuidade do projeto, pretende-se intensificar os estudos sobre neurociência, com o objetivo de adequação ao uso das metodologias computacionais com finalidades educativas. Ambas as áreas da Educação e da Computação serão beneficiadas, uma vez que estas metodologias possibilitarão o desenvolvimento de sistemas computacionais mais adequados ao processo de estruturação do pensamento dos usuários. Será possível o auxílio aos educadores no direcionamento da coleta de resultados de aprendizagem mais focados no objetivo pretendido, pois aproximações entre Ciência da Computação e Ciência Cognitiva já vem sendo traçadas por Saraiva e Argimon (2007). Os autores criam uma intersecção de visões onde a mente segue o funcionamento do computador e este busca imitar as funções da mente. Nesse contexto, a Ciência Cognitiva, por ser multidisciplinar, busca 
encontrar uma teoria unificada de cognição, integrando as diversas áreas do conhecimento em torno do estudo da mente.

O Pensamento Computacional, segundo Ribeiro (2012), oportuniza pensar e agir no sentido de como resolver problemas e quais equipamentos (computadores) corretos utilizar para esta resolução, desenvolvendo algoritmos que podem ser expressos em diferentes níveis de abstração.

Como proposições futuras o projeto de pesquisa envolverá maiores estudos sobre a Neurociência Cognitiva e contará com o desenvolvimento de um instrumento para coleta de dados melhor adaptado à pesquisa em ambiente computacional, para utilização em escolas com turmas mais numerosas. Esta intenção pretende redesenhar novas metodologias, com vistas a ampliar significativamente índices como o de $6 \%$, no caso desta pesquisa, considerando-se que 16,6\% representando uma pequena parte dos integrantes, apresentou resultados de aprendizagem condizentes apenas com o Nível IIA. Traçando um paralelo com a educação, em geral, um número de estudantes aquém do desejado vem obtendo sucesso na educação formal. A educação que oportuniza diferentes experiências como a ação dos estudantes sobre à uma linguagem de programação de computadores, apresenta maiores índices de sucesso.

Conclui-se, especificamente, que a base teórica fornecida pela Abstração Reflexionante e pela Neurociência Cognitiva são essenciais para que ocorram avanços do acompanhamento do processo de aprendizagem relacionada à evolução do Pensamento Computacional e que o fomento à inserção da programação nos currículos escolares de nível básico é urgente.

\section{REFERÊNCIAS}

CERVO, A. L.; BERVIAN, P. A.; SILVA, R. da. Metodologia científica. 6. ed. São Paulo: Prentice Hall, 2007. 162 p. ISBN 978-85-7605-047-6

CRUZ, M. E. J. K.; LUX, B.; HAETINGER, W.; HORN, F. Formação Prática do Licenciado em Computação para trabalho com Informática Educativa. In: XVIII Simpósio Brasileiro de Informática na Educação (SBIE), 2007, São Paulo. Anais do XVIII Simpósio Brasileiro de Informática na Educação. São Paulo: Sociedade Brasileira de Computação (SBC), 2007. p. 422-431.

CRUZ. Desenvolvimento e Comercialização de Kit de Robótica Educativa UniversidadeEmpresa. Anais do XXVIII Encontro Nacional de Engenharia de Produção. A integração de cadeias produtivas com a abordagem da manufatura sustentável, Rio de Janeiro, RJ, Brasil, 13 
a 16 de outubro de 2008 .

CRUZ. Formação de Licenciados em Computação no Brasil - Desenvolvimento e Utilização do ROBOKIT. Revista Revista Iberoamericana de Tecnologias el/Aprendizaje/Aprendizagem - IEEE, v.4, n. 3, 2009a. Disponível em: 〈http://webs.uvigo.es/cesei/RITA/200908/>. Acesso em: janeiro de 2014.

CRUZ. Controle de Kit de Robótica através de Laboratório Remoto pela Internet: uma Aplicação para a Formação Docente e para a Educação Básica. In: XX Simpósio Brasileiro de Informática na Educação (SBIE), 2009, Florianópolis. Anais do XVIII Simpósio Brasileiro de Informática na Educação. Florianópolis: Sociedade Brasileira de Computação (SBC), 17 a 20 de novembro de 2009 b.

DAMÁSIO, A. R. O erro de Descartes: emoção, razão e o cérebro humano. São Paulo: Companhia das Letras, 1996.

DAMÁSIO. A. R. O mistério da consciência: do corpo e das emoções ao conhecimento de si. São Paulo: Companhia das Letras, 2000.

KANDEL, E. R.; SCHARTZ, J. H.; JESSEL, T. M. (Coord.). Fundamentos da neurociência e do comportamento. Rio de Janeiro: Prentice-Hall do Brasil, 1997.

MATLIN, M. W. (2004). Psicologia cognitiva. 5. ed. Rio de Janeiro: LTC.

NUNES, D. J. Computação ou informática? Jornal da Ciência. Disponível em: Acesso em: 13 de Fevereiro de 2014.

PIAGET, J. A representação do mundo na criança: com o concurso de onze colaboradores. Aparecida: Idéias \& Letras, 2005.

PIAGET, J. Abstração reflexionante: relações lógico-aritméticas e ordem das relações espaciais. Porto Alegre: Artes Médicas, 1995.

PINKER, S. (1998). Como a mente funciona. São Paulo: Companhia das Letras.

RIBEIRO, L. O que é Computação? Da Máquina de Turing ao Pensamento Computacional. Instituto de Informática-Universidade Federal do Rio Grande do Sul - UFRGS, 2012.

Disponível em:

<http://inf.ufrgs.br/index.php?option=com_content\&view=article\&id=1319\%3Ao-que-ecomputacao-da-maquina-de-turing-ao-pensamento-computacional-leila-ribeiro-predio-

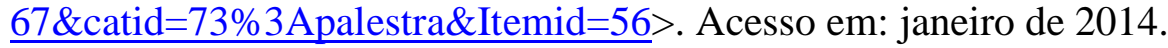

SARAIVA, C. A. E. e ARGIMON, I. de L. Ciência da computação e ciência cognitiva: um paralelo de Semelhanças. Ciências \& Cognição, v. 12, p. 150-155, 2007. Disponível em: <http://www.cienciasecognicao.org>. Acesso: maio de 2013.

TABACOW, L. S. Por dentro do cérebro do aprendiz: uma nova abordagem no processo educacional com a neurociência cognitiva: um guia para pais e educadores. Sorocaba: O Clássico, 2007. 